\title{
Role of Plasma Surface Treatments on Wetting and Adhesion
}

\author{
Rory Wolf ${ }^{1}$, Amelia Carolina Sparavigna ${ }^{2}$ \\ ${ }^{1}$ Enercon Industries Corporation, Menomonee Falls, Wisconsin, USA \\ ${ }^{2}$ Dipartimento di Fisica, Politencico di Torino, Torino, Italy \\ E-mail: rwolf@enerconmail.com, amelia.sparavigna@polito.it \\ Received December 9, 2009; revised February 13, 2010; accepted February 18, 2010
}

\begin{abstract}
There are many current and emerging wetting and adhesion issues which require an additional surface processing to enhance interfacial surface properties. Materials which are non-polar, such as polymers, have low surface energy and therefore typically require surface treatment to promote wetting of inks and coating. One way of increasing surface energy and reactivity is to bombard a polymer surface with atmospheric plasma. When the ionized gas is discharged on the polymer, effects of ablation, crosslinking and activation are produced on its surface. In this paper we will analyse the role of plasma and its use in increasing the surface energy to achieve wettability and improve adhesion of polymeric surfaces.
\end{abstract}

\section{Keywords: Atmospheric Plasma, Surface Treatments, Polymeric Films}

\section{Introduction}

The packaging industry is experiencing a technological revolution aimed at increasing consumer convenience and protection, and delivering new solutions for manufacturing and the distribution chain. Many wetting and adhesion issues are therefore emerging, which require an additional surface processing to promote wetting of inks and coating and enhance adhesion with these and other substances, to obtain high performance composited structures with polymeric and metallic foils.

Adhesion is a manifestation of attractive forces among atoms. There is a general agreement on the fact that attractions due to hydrogen and Van der Walls bonding are sufficient to produce an adhesive joint between polymers [1]. When adhesion of polymers with other materials such as metallic foils is necessary, chemical reactions at the interface leading to covalent chemical bonds are required $[1,2]$. This means that it is necessary the presence of highly reactive functional groups at the surface.

One way of increasing surface energy and reactivity is to treat the surface of the polymeric substrate with atmospheric plasma, which is an ionized gas at atmospheric pressure. When plasma gas is discharged on the polymer, effects of ablation, crosslinking and activation are produced on its surface.

While corona, flame and priming surface pre-treatments have been traditionally used in preparing finished flexible packaging structures for graphic and coating enhancements, the technology of atmospheric plasma treaters is giving evidence of performance benefits in increasing wettability and adhesion of polymeric surfaces [2-8]. More recently, experiments with plasma treatment coupled with grafting copolymerization [9-11], and used to enhance vapour depositions, show evidence for clear barrier deposition. These barriers, according to their relative deposition procedures, displayed hydrophobic, hydrophilic, anti-fog, biocide and anti-bacterial results. These very recent plasma treatments can have several applications for medical polymers $[12,13]$.

The atmospheric plasma treatment (APT) process was developed for treating/functionalizing a wide range of materials and has advantages over the presently used technologies of corona, flame, and priming treatments for flexible packaging applications. The APT system allows the creation of uniform and homogenous highdensity plasma at atmospheric pressure and at low temperature, utilising a broad range of inert and reactive gases $[14,15]$. Here we will discuss the role of APT in increasing the energy of polymeric surface. The energy produces a consequent increase in wettability and adhesion.

\section{Atmospheric Plasma Treatment Processes}

If a substrate has a low surface energy, its wettability is poor and coating adhesion very scarce, and then needs a surface treatment to increase energy. There are several 
methods for non-polar substrates. Those that can be configured as in-line treatment systems serve as economical alternatives to chemical primers, batch-treating processes, speciality coatings, and adhesives. These in-line treatments are mainly corona, flame and atmospheric plasma. A flame system creates a flame plasma field when flammable gas and air are combined and combusted to form a blue flame [8]. Brief exposures to particles within the flame affect the distribution and density of electrons on the substrate and polarise surface molecules through oxidation. This method also deposits other functional chemical groups that further promote ink wetting and adhesion.

A corona treating system is designed to increase the surface energy of plastic films, foils and paper in order to improve wettability and adhesion [16]. A corona treating system consists of two major components: the power supply and the treater station. The treater applies the power to the substrate, through an air gap, via a pair of electrodes, one at high potential and a roll supporting the material at the ground potential. Only the side of the material facing the high potential electrode should show an increase in surface energy.

Much like a corona discharge, an atmospheric plasma discharge is generated at atmospheric pressure. Instead of using air, this method relies on other gases that deposit specific chemical groups on the substrate surface to improve its surface energy and adhesion characteristics.

APT process acts on material surfaces in a way which is similar to the vacuum plasma treatment process. APT production equipment testing has been performed for the treatment of several materials, including the most utilised in applications such as polypropylene, polyethylene, polyester, polyamide, and polytetrafluoroethylene. The surface energies of the treated materials increased substantially, without any backside treatment or pin-holing [17], thereby enhancing wettability, printability, and adhesion properties.

The APT process consists of exposing a polymer to a low-temperature, high density glow discharge. Figure 1 shows the air gap, the dimension of which can range from $1.0 \mathrm{~mm}$ to $2.5 \mathrm{~mm}$, between the electrodes occupied by the glowing reacted gas. Polymeric films run above the lower electrode connected to the ground.

The resulting plasma is a partially ionised gas consisting of large concentrations of excited atomic, molecular, ionic, and free-radical species. Excitation of the gas molecules is accomplished by subjecting the gas, which is delivered within an open station design, to an electric field, typically at high frequency. Free electrons gain energy from the imposed high frequency electric field, colliding with neutral gas molecules and transferring energy, dissociating the molecules to form numerous reactive species. It is the interaction of these excited spe-

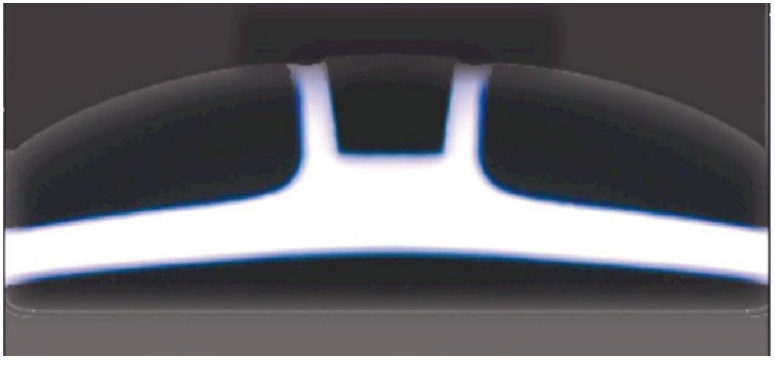

Figure 1. Air gap between the electrodes of Enercon plasma treater, occupied by the glowing gas. Gap dimension can range from $1 \mathrm{~mm}$ to $2.5 \mathrm{~mm}$. Polymeric films run above the lower electrode connected to the ground.

cies with solid surfaces placed in opposition to the plasma that results in the chemical and physical modification of the material surface.

The effect of plasma on a given material is determined by the chemistry of the reactions between the surface and the reactive species present in the plasma. At the low exposure energies typically used for surface treatment, the plasma surface interactions only change the surface of the material; the effects are confined to a region only several molecular layers deep and do not change the bulk properties of the substrate.

The resulting surface changes depend on the composition of the surface and the gas used. Gases or mixtures of gases, used for plasma treatment of polymers can include $\mathrm{N}, \mathrm{Ar}, \mathrm{O}_{2}, \mathrm{He}$, nitrous oxide, water vapour, carbon dioxide, methane, ammonia, and others. Each gas produces a unique plasma composition and results in different surface properties. For example, the surface energy can be increased very quickly and effectively by plasma-induced oxidation. Depending on the chemistry of the polymer and the source gases, substitution of molecular moieties into the surface can make polymers very wettable. The specific type of substituted atoms or groups determines the specific surface potential.

\section{Ablation, Crosslinking and Activation}

For any gas composition, three surface processes simultaneously alter flexible packaging substrates, with the extent of each depending on the chemistry and process variables: ablation, crosslinking, and activation [18].

In the ablation process, the bombardment of the polymer surface by free radicals, electrons, ions and radiation breaks the covalent bonds of the polymer backbone, resulting in lower-molecular-weight polymer chains. As long molecular components become shorter, the volatile oligomer and monomer by-products vaporise off (ablate) and are swept away with exhaust.

Crosslinking is done with an inert process gas (Ar or $\mathrm{He}$ ). The bond breaking occurs on the polymer surface. But since there are no free-radical scavengers, it can 
form a bond with a nearby free radical on a different chain (crosslink).

Activation is a process where surface polymer functional groups are replaced with different atoms or chemical groups from the plasma. As with ablation, surface exposure to energetic species abstracts hydrogen or breaks the backbone of the polymer, creating free radicals. In addition, plasma contains very high-energy UV radiation. This UV energy creates additional free radicals on the polymer surface. Free radicals, which are thermodynamically unstable, quickly react with the polymer backbone itself or with other free-radical species present at the surface to form stable covalently bonded atoms or more complex groups.

Application of atmospheric plasma to finished films has been theorised and practised to provide specific functionality to the base film substrate adequate for improved adhesion relative to the corona treatment process $[19,20]$. Since atmospheric plasma contains highly reactive species within the high density plasma at atmospheric pressure, it is proven to significantly increase surface area and to create polar groups on the surface of polymers so that strong covalent bonding between the substrate and its interface (i.e., inks, coatings, and adhesives) takes place.

It was therefore interesting to modify the polymeric surface in atmospheric plasma and study any change, relative to the untreated surface, of atomic bonding. This surface characterization of untreated and treated polymeric films can be obtained using the Electron Spectroscopy for Chemical Analysis (ESCA). Surface analysis by ESCA (also known as XPS, X-ray photoelectron spectroscopy) is accomplished by irradiating a sample with soft X-rays and analyzing the energy of ejected electrons. When the sample is irradiated, the resulting photoelectrons have energy depending on their original binding energy. A typical ESCA spectrum is a plot of the number of electrons detected per unit of time versus their binding energy. Each element produces a characteristic set of peaks at specific binding energy values, corresponding to the electron configurations within atoms. The number of detected electrons in each peak is directly related to the amount of element within the area irradiated. ESCA then could be used to identify and determine the concentration of the elements on the surface [21].

Figure 2 shows the C1s ESCA spectra for an untreated PET film and PET films after $\mathrm{Ar} / \mathrm{O}_{2}$ plasma treatments. The X-ray photoelectron spectroscopy analysis suggests a modification of the surface when subjected to a plasma treatment. In particular, comparing $\mathrm{C} 1 \mathrm{~s}$ spectra of untreated and treated samples, we note that number of functional groups is clearly changed. Let us remember that $\mathrm{C} 1 \mathrm{~s}$ spectrum is coming from three different groups of carbon atoms: the relative intensities of the structures at $289,286.5$ and $285 \mathrm{eV}$ corresponds to $\mathrm{C}$ atoms in $\mathrm{O}-\mathrm{C}=\mathrm{O}$, $\mathrm{C}-\mathrm{C}-\mathrm{O}$ and $\mathrm{C}-\mathrm{C}-\mathrm{C}$ bonding positions.

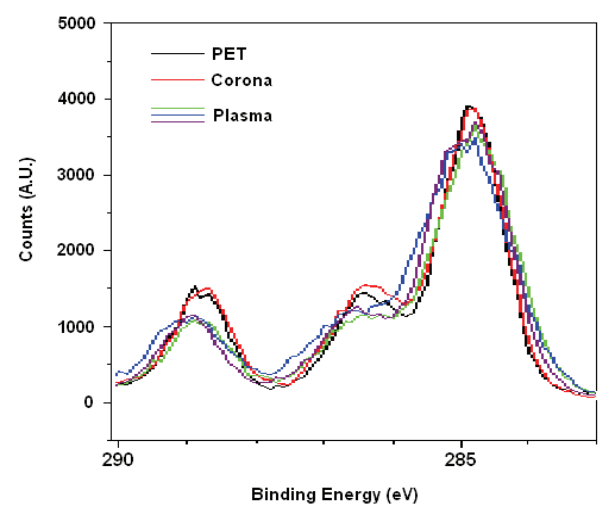

Figure 2. C1s ESCA spectra as a function of binding energies for untreated PET film and PET films after corona and $\mathrm{Ar} / \mathrm{O}_{2}$ plasma treatments.

Figure 2 is also showing the spectrum of a corona treated sample. The intensity of the highest peak, the aromatic peak, decreases after plasma treatment, whereas the corona treatment seems to leave it unchanged. The relative intensity of second peak at $286.5 \mathrm{eV}$ is reduced in the case of plasma treated samples. The broadening combines this second peak with the first one (green, blue and purple lines). In the case of the corona treated sample (red line), the second peak is strongly increased. The third peak at $289 \mathrm{eV}$ does not change after corona treatment, but it is reduced and broadened by plasma treatments.

Peak broadening corresponds to an enhancement of the number of functional groups on the polymeric surface. We note a different behaviour of spectra obtained from samples after corona and $\mathrm{Ar} / \mathrm{O}_{2}$ plasma treatments. This could be due to the fact that treatments carried out in plasma with inert gases introduce oxygen moieties onto the polymer surface during a post plasma exposure of samples to atmosphere [22,23]. An Ar containing plasma treatment causes the breakage of some bonds, leading to the formation of the carbon radicals and crosskinking effects. The surface crosslinking can compete with the oxidation process, influencing the number of covalently bond groups introduced by plasma treatment [2,24 and references therein]. This explains the difference of plasma and corona results in ESCA. A detailed discussion of the mechanisms which produce the surface modifications of $\mathrm{PET}$ in $\mathrm{Ar} / \mathrm{O}_{2}$ plasma treatments is proposed in Ref. [25].

\section{Surface Energy and Wetting}

The surface energy of a substrate can be evaluated by wetting it with liquids which have well-known surface tensions. The measurement can be obtained through the contact angle of a pure liquid droplet on the solid substrate, for instance by means of the sessile drop method. In this method, a syringe pointed vertically down onto the 
sample surface deposits the liquid droplet. The liquid used is referred to as the probe liquid, and the use of several different probe liquids is required. This method is relatively straightforward. If the solid surface under investigation is large enough, multiple droplets can be deposited in various locations on the sample to determine possible heterogeneity.

To determine the surface free energy of a polymer with its polar and dispersive portions, the contact angle is measured with a number of test liquids and evaluated according, for instance to Wu's method [26]. At least two test liquids with known surface tension and its polar and dispersive contributions are required. Each additional liquid will increase the accuracy of the estimation. Untreated polypropylene films, for instance, have a very low polar portion of the surface energy. After flame and corona treatments, the polar part is strongly increased: experimental results are shown in Table 1.

There are other methods to determine the surface tensions (Du Noüy Ring method, Wilhelmy method, Spinning and Pendant Drop methods). From a practical point of view, when it is necessary to evaluate the surface energy obtained with corona and plasma treaters, placed on-line in converter devices for printing and lamination, the Dyne Solution method prevails [27]. This is the preferred method to determine the surface energy of polymeric films treated with a specific device and is vital for converters.

If a substrate has a low surface energy, its wettability is poor and coating adhesion very scarce with bad final results after printing. In-line treatments, such as corona, flame and atmospheric plasma, have a profound impact on the polar component of surface energy, increasing it and modifying the surface functionalities of materials $[19,20]$. These materials include polymers such as polyester, polyethylene and polypropylene.

In many industries, high surface energy (above the value of $50 \mathrm{mN} / \mathrm{m}$ ) is required for satisfactory wettability, adhesion and printability. Figure 3 shows linear trends of the surface energy of polyester (PET), polyethylene (PE) and polypropylene (PP) films, treated by Enercon atmospheric plasma treatment technology, with increasing power. The gas chemistries applied to form a uniform

Table 1. Surface energies and their dispersive and polar contributions in $(\mathrm{mN} / \mathrm{m})$, evaluated according to Wu's method (see Ref. [26]).

\begin{tabular}{llll}
\hline Film & $\begin{array}{l}\text { Surface } \\
\text { Energy } \\
(\mathbf{m N} / \mathbf{m})\end{array}$ & Dispersive & Polar \\
& 29.98 & 29.95 & $\mathbf{( m )})$ \\
\hline Untreated PP & 38.50 & 30.19 & 0.03 \\
Corona treated PP & 39.19 & 30.20 & 8.31 \\
Flame treated PP & & 8.99 \\
\hline
\end{tabular}

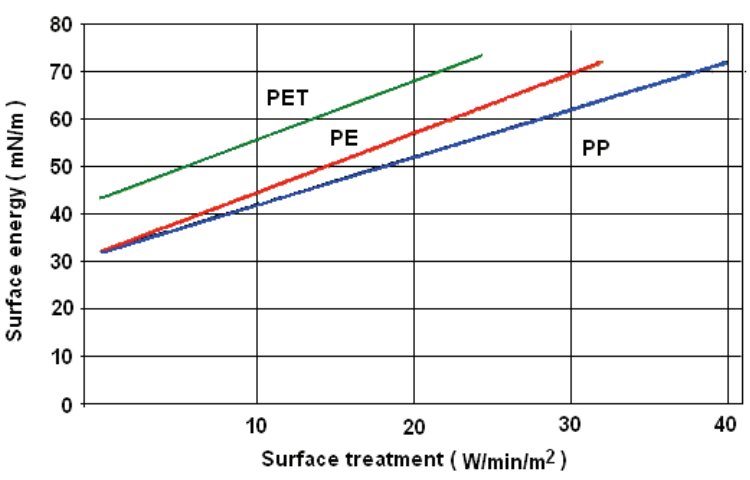

Figure 3. Linear trends of surface energies of polyester, polyethylene and polypropylene films, treated by Enercon atmospheric plasma treatment technology as a function of the increasing treatment power.

high density atmospheric plasma included Ar with small percentages of reactive gases. The gas chemistries typically applied to each of these materials are as follows: for $\mathrm{PP}-\mathrm{Ar} / \mathrm{O}_{2}$, for PE-Ar $/ \mathrm{O}_{2} /$ Acetylene and for PET-Ar $/ \mathrm{O}_{2}$.

The linear trends in Figure 3 were obtained from measurements of surface energies by means of Dyne Solutions during on-line processing. As can be seen with regard to PET, its base (untreated) surface energy is approximately of $43 \mathrm{mN} / \mathrm{m}$. This is high, relative to polyolefins, since polyester is synthesised with purified terephthalic acid (PTA) or its dimethyl ester dimethyl terephthalate (DMT) and monoethylene glycol (MEG), all fairly polar components. As obtained from Dyne Solutions measurements, $6 \mathrm{~W} / \mathrm{min} / \mathrm{m}^{2}$ are required to move PET surface energy to a value of $50 \mathrm{mN} / \mathrm{m}$, and $14 \mathrm{~W} / \mathrm{min} / \mathrm{m}^{2}$ to achieve a value of $60 \mathrm{mN} / \mathrm{m}$.

$\mathrm{PE}$ is a polymerised ethylene resin, containing both carbon and hydrogen, with a resident surface tension of around $31 \mathrm{mN} / \mathrm{m}$ and very low polarity. Although a power density of $14.5 \mathrm{~W} / \mathrm{min} / \mathrm{m}^{2}$ is generally required to achieve $50 \mathrm{mN} / \mathrm{m}$, this is significantly less than the average corona discharge requirement of approximately $30 \mathrm{~W} / \mathrm{min} / \mathrm{m}^{2}$. PP is a thermoplastic polyolefin with a relatively high level of crystallinity and very low polarity. It is formed by polymerising propylene with suitable catalysts, such as aluminum alkyl and titanium tetrachloride. Its resident surface tension is about $31 \mathrm{mN} / \mathrm{m}$, again due to its inertness. Although its chain mobility can be considerably less than other polyolefins, atmospheric plasma can raise its surface tension to over $50 \mathrm{mN} / \mathrm{m}$ with treatment at $18 \mathrm{~W} / \mathrm{min} / \mathrm{m}^{2}$. Again, this is significantly below the corona discharge dosage of nearly $50 \mathrm{~W} / \mathrm{min} / \mathrm{m}^{2}$, typically required.

\section{Experiments on Adhesion}

We compared also the effects of surface treatment with plasma and corona on the printing adhesion. As in the evaluation of the surface energy trend, the trial runs were 
performed on Enercon's Bare Roll Corona Treatment Station, as well as its Plasma3 ${ }^{\mathrm{TM}}$ Atmospheric Plasma Treatment System, resident on its same pilot line. For all runs, a PVDC-coated polyester film was post-treated and then printed with aqueous ink using a laser-engraved anilox roll. The printed image provided solid (100\%) ink coverage. The calculated ink transfer (thickness) to the film, based upon the cell volume anilox roll, is approximately 1.9 microns. The printed web drying temperature occurred at $121^{\circ} \mathrm{C}$, with a web temperature of $77^{\circ} \mathrm{C}$. The levels of power treatment used in this design and of the surface energy after treatment are shown in Table 2.

A friction/peel testing equipment conforming to test standards (ASTM-D1894,-D4521,-D3330, TAPPI-T816, DIN-53375, BS-2782 and PSTC-1, 3, 4, 5) was used for testing the printed polyester film. The testing protocol employed twenty measured peel iterations for unprinted and printed samples, each of which were corona and APT-treated. Results in terms of the averages of these iterations are given in Table 3.

The peel adhesion data indicated that at a power density of $10 \mathrm{~W} / \mathrm{min} / \mathrm{m}^{2}$, printed APT-treated PET surpassed the peel adhesion results registered by printed corona-treated polyester. Moreover, the decrease in ink peel adhesion between unprinted and printed corona-treated base material, compared to the less than a $4 \%$ decline in ink peel adhesion between unprinted and printed APT-treated base material, suggests that the for-

Table 2. Variable levels utilised in the experimental design.

\begin{tabular}{llll}
\hline Variable & APT & Corona & Control \\
\hline Substrate & $23 \mu \mathrm{m}$ PET & $23 \mu \mathrm{m}$ PET & $23 \mu \mathrm{m}$ PET \\
Pretreatment & None & None & None \\
Power Density & $10 \mathrm{~W} / \mathrm{min} / \mathrm{m}^{2}$ & $10 \mathrm{~W} / \mathrm{min} / \mathrm{m}^{2}$ & None \\
Post-Treatment & $54 \mathrm{mN} / \mathrm{m}$ & $46 \mathrm{mN} / \mathrm{m}$ & $40 \mathrm{mN} / \mathrm{m}$ \\
Treatment & Helium $/ \mathrm{O}_{2}$ & None & None \\
Chemistry & Water-based & Water-based & None \\
Ink Chemistry & & & \\
\hline
\end{tabular}

Table 3. Average peel adhesion for printed and unprinted PET film after power treatment of $10 \mathrm{~W} / \mathrm{min} / \mathrm{m}^{2}$.

\begin{tabular}{lll}
\hline Sample & $\begin{array}{l}\text { Average } \\
(\mathbf{N} / \mathbf{c m})\end{array}$ \\
\hline Corona treated, unprinted & 1.28 \\
Corona treated, printed & 0.98 \\
Plasma treated, unprinted & 1.80 \\
Plasma treated, printed & 1.74 \\
\hline
\end{tabular}

Table 4. Average peel adhesion for printed and unprinted PET film for a surface energy of $46 \mathrm{mN} / \mathrm{m}$.

\begin{tabular}{lll}
\hline Sample & $\begin{array}{l}\text { Average } \\
(\mathbf{N} / \mathbf{c m})\end{array}$ & Peel Adhesion \\
\hline Corona treated, unprinted & 1.28 & \\
Corona treated, printed & 0.98 & \\
Plasma treated, unprinted & 1.60 \\
Plasma treated, printed & 1.45 \\
\hline
\end{tabular}

mation of strong covalent atomic bonds on a cleaned and uniform, homogeneously micro-etched surface may account for improved anchorage of inks.

To determine the impact on peel adhesion under conditions where the surface tension created by both corona and APT were the same, the protocols were repeated and data reported in Table 4. This condition was established by reducing the power density of APT to $7 \mathrm{~W} / \mathrm{min} / \mathrm{m}^{2}$, to achieve $46 \mathrm{mN} / \mathrm{m}$. This set of peel adhesion data indicates that at a surface tension level of $46 \mathrm{mN} / \mathrm{m}$ printed APT-treated polyester maintained a significantly higher peel adhesion performance over printed corona-treated polyester.

The analysis identified that untreated flexible packaging grade polyester film which was post-treated with the APT process exhibited high levels of peel adhesion relative to the corona post-treated polyester at a power density of $10 \mathrm{~W} / \mathrm{min} / \mathrm{m}^{2}$. When post-treatment surface tension was equalized between APT and corona at $46 \mathrm{mN} / \mathrm{m}$, the APT treatment process continued to promote strong ink anchorage relative to corona by approximately fifty percent.

It is not easy a comparison with experimental data obtained from other research groups, because experimental set-up designs are different. We observed an agreement with data on the strength adhesion increase, after air plasma treatment, as reported in Ref. 23. This is a quite interest result, because we are using industrial treatment systems. Other data on PET, such as those in Ref. [28], were obtained in rather different conditions and impossible to compare. These data are in any case reporting an increase of adhesion after plasma treatment.

A different peel strength obtained after plasma or corona treatment is caused by several factors. It is usually believed that it is the creation of a wettable polar surface responsible for the increase of strength. As observed in [23], this is a sufficient condition for forming strong joints. A necessary condition is to clean the surface and remove weak boundary layers from it. The plasma cleaning is well-known and widely used to achieve clean surfaces. Moreover, as seen from AFM photographs, the surface assumes, after a plasma treatment, a roughness which increases the effective surface area suitable for adhesion [23]. Thus, adhesion will be facilitated by all these factors.

\section{Conclusions}

In this paper we discussed the atmospheric plasma treatment of polymeric surfaces. Let us note that the plasma treatment system can be operated at low temperatures and at atmospheric pressure, thereby eliminating the need for any vacuum chambers or pumps. Atmospheric plasma provides then the advantages which plasma technology has over the existing technologies for surface treatment of polymers, without additional costs. The systems we used for investigation on wetting and 
adhesion are commercial devices, then confirming the possibility of plasma to be used in industrial converting systems.

The surface energies of polymers treated by atmospheric plasma systems have been shown to increase substantially, thereby significantly enhancing the wettability, printability and adhesion properties. The peel tests indicate that the APT process can affect better ink adhesion than corona treatment. Our analysis on adhesion provided evidence that flexible packaging converters utilizing aqueous inks on polyester-based structures may experience improvements in ink adhesion by employing APT-based surface treatment systems instead of corona treaters.

\section{References}

[1] D. K. Owens and R. C. Wendt, "Estimation of the Surface Free Energy of Polymers," Journal of Applied Polymer Science, Vol. 13, No. 18, 1969, pp. 1741-1747.

[2] Y. M. Chung, M. J. Jung, J. G. Han, M. W. Lee and Y. M. Kim, "Atmospheric RF Plasma Effects on the Film Adhesion Property," Thin Solid Films, Vol. 447-448, 2004, pp. 354-358.

[3] N. Inagaki, "Plasma Surface Modification and Plasma Polymerization," CRC Press, Boca Raton, 1996.

[4] M. A. Lieberman and A. J. Lichtenberg, "Principles of Plasma Discharges and Materials Processing," John and Wiley, Hoboken, 1994.

[5] J. Reece Roth, "Industrial Plasma Engineering," Institute of Physics, 1995.

[6] M. Sugawara, "Plasma Etching," Oxford Science, Oxford, 1998.

[7] A. Yializis, S. A. Pirzada and W. Decker, "A Novel Atmospheric Plasma System for Polymer Surface Treatment," In: K. L. Mittal Ed., Polymer Surface Modification: Relevance to Adhesion, VSP, Utrecht, The Nederland, 2000, pp. 65-76.

[8] H. T. Lindland, "Flame Surface Treatment," In: D. Satas and A. A. Tracton Eds., Coatings Technology Handbook, Marcel Dekker, New York, 2001.

[9] R. Wolf, A. Sparavigna and R. Ellwanger, "Modifying the Surface Features-IV-Clear Barrier Films," Converter: Flessibili, Carta, Cartone, Vol. 67, 2007, pp. 72-85.

[10] R. Wolf and A. Sparavigna, "Modifying the Surface Features," Coating, Vol. 41, 2008, pp. 24-26.

[11] N. K. Cuong, N. Saeki, S. Kataoka and S. Yoshikawa, "Hydrophilic Improvement of PET Fabrics Using PlasmaInduced Graft Polymerization," Hyomen Kagaku, Vol. 23, No. 4, 2002, pp. 202-208.

[12] Rapra Technology Ltd, "The Medical Polymers," 5th International Conference Focusing on Polymers Used in the Medical Industry, Cologne, Germany, June 6-7, 2006.

[13] L. W. McKeen, "Fluorinated Coatings and Finishes Handbook: The Definitive User's Guide and Databook,"
William Andrew Inc., New York, 2006.

[14] J. Reece Roth, S. Nourgostar and T. A. Bonds, "The One Atmosphere Uniform Glow Discharge Plasma (OAUGDP)," IEEE Transactions on Plasma Science, Vol. 35, No. 2, 2007, pp. 233-250.

[15] J. Reece Roth, "Potential Industrial Applications of the One Atmosphere Uniform Glow Discharge Plasma Operating in Ambient Air," Physics of Plasmas, Vol. 12, No. 5, 2005, pp. 1-9.

[16] R. A. Wolf, "Corona Treatment: A Process Overview," IDS Packaging, 2007.

[17] R. Wolf, A. Sparavigna and E. Descrovi, "Hidden Problems in Surface Treatments-I-Pinholing," Converter: Flessibili, Carta, Cartone, Vol. 70, 2008, pp. 96-104.

[18] R. D'Agostino, "Plasma Deposition, Treatment, and Etching of Polymers: The Treatment and Etching of Polymers," Academic Press, Boston, 1990.

[19] S. Nowak and O. M. Kûttel, "Plasma Treatment of Polymers for Improved Adhesion Properties," Materials Science Forum, Vol. 140-142, 1993, pp. 705-726.

[20] F. Massines and G. Gouda, "A Comparison of Polypropylene-Surface Treatment by Filamentary, Homogeneous and Glow Discharges in Helium at Atmospheric Pressure," Journal of Physics D: Applied Physics, Vol. 31, No. 24, 1998, pp. 3411-3420.

[21] J. F. Moulder, W. F. Stickle, P. E. Sobol and K. D. Bomben, "Handbook of X-ray Photoelectron Spectroscopy," Perkin-Elmer Corp., Eden Prairie, USA, 1992.

[22] B. Gupta, J. Hilborn, C. H. Hollenstein, C. J. G. Plummer, R. Houriet and N. Xanthopoulos, "Surface Modification of Polyester Films by RF Plasma," Journal of Applied Polymer Science, Vol. 78, No. 5, 2000, pp. 1083-1091.

[23] R. R. Deshmukh and V. N. Bhat, "The Mmechanism of Adhesion and Printability of Plasma Processed PET Films," Materisls Research Innovations, Vol. 7, No. 5, 2003, pp. 283-290.

[24] H. Iwata, H. Kishida, M. Suzuki, Y. Hata and Y. Ikada, "Oxidation of Polyethylene Surface by Corona Discharge and the Subsequent Graft Polymerization," Journal of Polymer Science: Polymer Chemistry, Vol. 26, No. 12, 1988, pp. 3309-3322.

[25] N. Inagaki, S. Tasaka, K. Narushima and H. Kobayashi, "Surface Modification of PET Films by Pulsed Argon Plasma," Journal of Applied Polymer Science, Vol. 85, No. 14, 2002, pp. 2845-2852.

[26] S. Wu, "Polymer Interface and Adhesion," CRC, New York, 1982.

[27] K. L. Mittal, "Contact Angle, Wettability and Adhesion, American Chemical Society Division of Colloid and Surface Chemistry, VSP," Utrecht, The Nederland, 1993.

[28] J. M. Shenton, M. C. Lovell-Hoare and G. C. Stevens, "Adhesion Enhancement of Polymer Surfaces by Atmospheric Plasma Treatment," Journal of Physics D: Applied Physics, Vol. 34, No. 18, 2001, pp. 2754-2760. 